This copy is for personal use, the final version will be publisehd in the proceedings of the 2013 International Conference on $3 D$ Vision (3DV 2013) (c) IEEE 2013

\title{
A Study of Point Cloud Registration with Probability Product Kernel Functions
}

\author{
Hanchen Xiong Sandor Szedmak Justus Piater \\ Institute of Computer Science, University of Innsbruck \\ Technikerstr.21a A-6020, Innsbruck, Austria \\ \{hanchen.xiong, sandor.szedmak, justus.piater\}@uibk.ac.at
}

\begin{abstract}
$3 D$ point cloud registration is an essential problem in $3 D$ object and scene understanding. In many realistic circumstances, however, because of noise during data acquisition and large motion between two point clouds, most existing approaches can hardly work satisfactorily without good initial alignment or manually marked correspondences. Inspired by the popular kernel methods in machine learning community, this paper puts forward a general point cloud registration framework by constructing kernel functions over 3D point clouds. More specifically, Gaussian mixtures based on the point clouds are established and probability product kernel functions are exploited for the registration. To enhance the generality of the framework, $S E(3)$ on-manifold optimization scheme is employed to compute the optimal motion. Experimental results show that our registration framework works robustly when many outliers are presented and motion between point clouds is relatively large, and compares favorably to related methods.
\end{abstract}

\section{Introduction}

The past two decades have witnessed great development in 3D point cloud acquisition and usage, as increasingly more state-of-the-art stereo vision reconstruction algorithms were developed and affordable range-finder devices emerged. However, there still exist many obstacles to full exploitation of 3D point clouds, among which 3D registration plays a fundamental role. Simply speaking, 3D point cloud registration is the problem of moving a model point cloud $\mathcal{M}$ to achieve the best possible alignment with a fixed scene point cloud $\mathcal{S}$ by minimizing a certain distance function between them. Mathematically, the motion $\mathbf{P}$ from $\mathcal{M}$ to $\mathcal{S}$ is a rigid transformation in $\mathbb{R}^{3}$, which is composed of a 3D rotation $\mathbf{R}$ and a translation t. Given $f$ as a distance function between two point clouds, the problem can be formulated as $\left\{\mathbf{R}^{*}, \mathbf{t}^{*}\right\}=\arg \min _{\mathbf{R}, \mathbf{t}} f(\mathcal{M}, \mathcal{S} ; \mathbf{R}, \mathbf{t})$. One can easily form $f$ as the sum of squared distances between all corresponding points from two clouds if the correspondence information is provided. Unfortunately, however, there is no existing method which can find the perfectly accurate correspondence between two point clouds. Iterative Closest Point (ICP) [1], the most popular method for 3D registration so far, assumes correspondence based on the closest distance criterion and then computes the transformation that best aligns the putative correspondences. Two steps are implemented alternately and iteratively so that computing either of them will improve the other.

Different from the iterative method, our algorithm avoids point-wise correspondence search. Instead, we consider point clouds in their entirety, and compute them as setformat data in kernel method. In other words, the distance function $f$ is formulated at the cloud level. First, continuous parametric probabilistic density functions (PDFs) are established as the representations of point clouds by applying kernel density estimation (KDE) with Gaussian kernels. Then, with KDE representations, an expected likelihood kernel function is employed to compute the affinity between two distributions, which corresponds to the similarity between two point clouds. Finally, the optimal pose is computed to maximize the kernel function, which is equivalent to minimizing the distance function $f$ between two point clouds.

The key contributions of our work can be summarized in two points: First, we express 3D point cloud registration in a kernel method framework with a special case study on probability product kernel functions. Although the scope of study in this paper is limited, it can indicate a new perspective of the registration problem with more potential kernel functions explored and studied in the future. Secondly, we exploit the $S E(3)$ on-manifold optimization scheme to provide an elegant solution to compute the optimal motion. Since the generality of kernel method and $S E(N)$ optimization, the resulting registration algorithm can be easily extended to any dimension cases (although we only concern 3D case here). 


\section{Related Work}

Since originally proposed [1], ICP and its variants almost dominate the research literature of 3D point cloud registration. As briefly mentioned in section 1, at each iteration of ICP, corresponding pairs are assumed to be found according to the nearest-neighbor criterion, based on which the optimal motion is subsequently computed. It has been realized that in practice this naive correspondence-searchassumption can fail if the motion between two point clouds is large or there exists a large amount of noise. Thus, many improvements to ICP methods were proposed. All ICP variants can be explained as iterative cycles of six steps [20]: (1) Select subsets of two points clouds: in most cases, all points are used. However, a subset can be randomly sampled to reduce the computational burden if the number of points is too large. (2) Match corresponding pairs: this is a key step which has drawn much attention to achieve improvement. The original version [1] matches pairs by closest distance. Many other studies tried to improve the correspondence accuracy by making use of more information such as normal vectors [7], curvature [23], and color [12]. Even some more sophisticated persistent point feature descriptors [21] were developed to find the exact matches. (3) Weight the corresponding pairs: Weighting alleviates the influence of poor correspondence matching. To each pair should be assigned a weight proportional to the likelihood of the correspondence, which can be computed as the compatibility of normals or colors [8]. (4) Reject pairs: To some degree, rejection is equivalent to weighting by only assigning binary weights $w=\{0,1\}$, so normal orientation and color compatibility can be computed in the same way; then a threshold is set to decide which pairs can be accepted. Some other geometric properties such as inter-point distance consistency and collinearity consistency [15] can be also used to filter out weak corresponding pairs. Other methods reject pairs in which two points do not bi-uniquely correspond to each other [24], or use weaker notions of consistency such as $\epsilon$-reciprocal correspondence [17]. (5) Compute an error metric: The error metric is usually designed as the sum of squared distances between corresponding points. To enhance the robustness to outliers of correspondences obtained from previous steps, Trimmed ICP was developed [4] by using trimmed squares, which is only composed of square distances with relatively small values. (6) Minimize the error metric: This is an optimization step with respect to $3 \mathrm{D}$ rotation and translation. Usually unit quaternion and the dual number quaternion methods [25] is used to compute rotation. Although there have been various improvements to ICP, its applicability is still limited to the scenario in which two point clouds can be fairly closely aligned in advance, and noise is low. Therefore, when used in practical applications, some manual assistance is usually required, which makes ICP methods barely suitable within fully automatic systems.

Besides the progress of ICP methods, some other novel approaches have been proposed from different perspectives. A notable contribution is spectral correspondence [3], where graphs are constructed based on point clouds, and the structural properties are extracted with spectral graph theory to find matching point patterns. Another influential work is SoftAssign [9], which establishes one-to-many correspondences with different weights, and the registration is solved as a joint optimization over the transformation and correspondence matrix.

Prior to our work, a related algorithm was proposed by Jian et al. in [11]. In Jian's method, each point cloud is similarly modelled globally and probabilistically with Gaussian mixtures, and the optimal motion is computed to minimize the L2 distance between corresponding Gaussian mixtures. However, we go beyond their work in several ways: first, instead of computing L2 distance between two fitted distributions, we consider the registration problem in a more general kernel-based framework, which results in more flexibility and extensibility. Secondly, in contrast to the identical and spherical covariance, we use more elaborately estimated bandwidths in KDE with Gaussian kernels to capture more local structural information of point clouds. Finally, instead of using unit quaternions, we exploit $S E(3)$ on-manifold optimization to achieve optimal motion estimation, which yields a rather general registration algorithm.

\section{Kernel Methods for Point Clouds}

Kernel methods have achieved remarkable success in many machine learning applications by enabling various linear models to exploit nonlinear data patterns, such as SVM and kernel PCA [22]. The kernel function is originally designed as a trick to efficiently compute the inner product (similarity) between the images of two data in a mapped (higher dimension) Hilbert feature space:

$$
K(x, z)=\langle\Phi(x), \Phi(z)\rangle
$$

where $x, z \in \chi$ and $\Phi(\cdot)$ is mapping function from $\chi$ to the feature space. A strength of the kernel method is that with properly designed kernel functions it can work with very general types of data, including strings, trees or graphs [22], without explicit feature maps. As a matter of fact, any symmetric similarity measurement between two data sets of certain types can be a kernel function as long as it satisfies positive semi-definiteness, and data types are implicitly embedded into an induced feature space. In this paper, we are focusing on point clouds, on which kernel methods can work in the exact same way to compute the similarity by mapping them into a Hilbert feature space. 


\subsection{Kernel methods for unordered sets of data}

Point clouds can be treated as unordered sets of vectors, on which different kernel functions have been already developed and studied [14, 16]. The basic methodology is to consider all elements in a set as i.i.d. samples from an underlying probability distribution, and thus the distance between two sets can be computed as the discrepancy between two corresponding distributions. One can model two sets probabilistically by fitting them to two distributions whose PDFs are of a certain parametric form, and then instead of developing new kernel functions on point clouds, existing kernel functions on probabilities can be directly used. Kondor and Jebara developed a Bhattacharyya kernel based on the Bhattacharyya affinity used in the statistics literature [14]:

$$
K_{B h a}(p, q)=\int \sqrt{p(x)} \sqrt{q(x)} \mathrm{d} x
$$

where $p$ and $q$ are two distributions. In [16], an expected likelihood kernel, which behaves as computing the expectation of either distribution under the other, is applied as

$$
K_{\exp }(p, q)=\int p(x) q(x) \mathrm{d} x
$$

Both the Bhattacharya kernel and the expected likelihood kernel are two special cases of probability product kernels [10] $K(p, q)=\int p(x)^{\rho} q(x)^{\rho} \mathrm{d} x$ with $\rho=0.5$ and $\rho=1$. Assuming point clouds $\mathcal{M}$ and $\mathcal{S}$ are fitted to two distributions $\phi$ and $\psi$ respectively, the kernel function on point clouds can be defined as

$$
K(\mathcal{M}, \mathcal{S})=\int \phi(x)^{\rho} \psi(x)^{\rho} \mathrm{d} x,
$$

and the induced Hilbert feature space corresponds to the fitted distribution (infinite-dimensional feature space) when $\rho=1$, and to the square root of the distribution when $\rho=0.5$.

\subsection{Probabilistic modeling of point clouds}

In order to apply(4), one has to model point clouds in a probabilistic form. Because (4) can be efficiently computed without explicit integration if the PDFs of the two distributions are in the exponential family, the Gaussian mixture model is usually used [14, 16]. In this paper, to simplify selecting the number of components, KDE (Kernel Density Estimation) with Gaussian kernel is applied to construct a Gaussian mixture with Gaussians constructed on all points. This approach has been followed in other successful work $[5,6,11]$ to establish a probabilistic form of point clouds. However, one weakness of KDE in this other work is that only identical and isotropic covariance is used to construct corresponding Gaussian kernels, which limits the representational power to exploit geometric details of

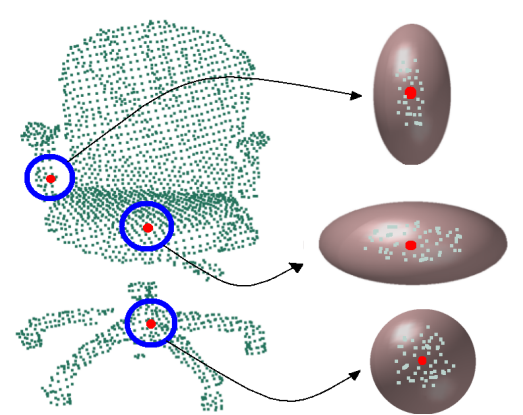

Figure 1. The covariance of the kernel associated with each point is locally determined from its neighbourhood and thus well captures local structure.

the point clouds. Therefore, in our algorithm, in order to capture the local structural information as much as possible, a full covariance is estimated with the neighboring region of each point (Figure 1). Given a model point cloud $\mathcal{M}=\left\{\lambda_{\mathcal{M}}^{(i)} \in \mathbb{R}^{3}\right\}_{i=1}^{m}$, a Gaussian kernel can be established with its mean equal to the 3D position of each point and covariance estimated with the surroundings of the point. Thus the KDE representation of the model point cloud can be written as

$$
\phi(x)=\frac{1}{m_{\mathcal{M}}} \sum_{i=1}^{m_{\mathcal{M}}} \mathcal{N}_{\mathbb{R}^{3}}\left(x ; \lambda_{\mathcal{M}}^{(i)}, \Sigma_{\mathcal{M}}^{(i)}\right)
$$

where $\mathcal{N}\left(x ; \lambda_{\mathcal{M}}^{(i)}, \Sigma_{\mathcal{M}}^{(i)}\right)$ denotes the normal distribution with mean $\lambda_{\mathcal{M}}^{(i)}$ and covariance $\Sigma_{\mathcal{M}}^{(i)}$, and $m_{\mathcal{M}}$ is the size of model point cloud. Similarly, the KDE representation of the scene point cloud $\mathcal{S}$ can be constructed by the same procedure.

\subsection{A kernel function on two point clouds}

To put all pieces together, a kernel function on point clouds can be formulated by substituting (5) into (4). For the sake of clarity in describing the framework, here we only study the case of expected likelihood kernel function ( $\rho=1$ ) although other kernel functions can be analogously derived and explored. Thus (4) can be rewritten as:

$$
\begin{aligned}
K(\mathcal{M}, \mathcal{S})=\frac{1}{m_{\mathcal{M}}} \frac{1}{m_{\mathcal{S}}} \sum_{i=1}^{m_{\mathcal{M}}} \sum_{j=1}^{m_{\mathcal{S}}} \mathcal{G}_{\mathcal{M}^{i} \mathcal{S}^{j}} \\
\mathcal{G}_{\mathcal{M}^{i} \mathcal{S}^{j}}=\int \mathcal{N}_{\mathbb{R}^{3}}\left(x ; \lambda_{\mathcal{M}}^{(i)}, \Sigma_{\mathcal{M}}^{(i)}\right) \mathcal{N}_{\mathbb{R}^{3}}\left(x ; \lambda_{\mathcal{S}}^{(j)}, \Sigma_{\mathcal{S}}^{(j)}\right) \mathrm{d} x \\
=\frac{1}{(2 \pi)^{3 / 2}\left|\Sigma^{*}\right|^{1 / 2}} \exp \left\{-\frac{1}{2} \lambda^{*^{\prime}} \Sigma^{*-1} \lambda^{*}\right\}
\end{aligned}
$$

where $|\cdot|$ denotes the determinant, and $\lambda^{*}=\lambda_{\mathcal{M}}^{(i)}-\lambda_{\mathcal{S}}^{(j)}$ and $\Sigma^{*}=\Sigma_{\mathcal{M}}^{(i)}+\Sigma_{\mathcal{S}}^{(j)}$ according to Gaussian identities [19]. 


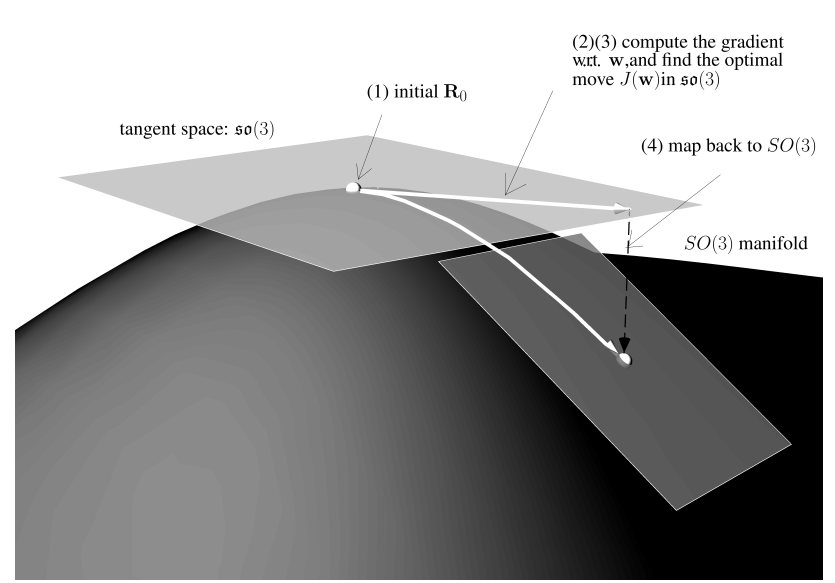

Figure 2. The $\mathrm{SO}(3)$ manifold and its optimization scheme: (1) start from a rotation matrix $\mathbf{R}_{0}$; (2) use equation (10) as the local parametrization of the manifold at point $\mathbf{R}_{0}$, and compute the gradient $\mathbf{g}$ with respect to $\mathbf{w}$; (3) compute the best move in $\mathfrak{s o}(3)$ by mapping $J(\mathbf{w})$; (4) map back to $S O(3): \mathbf{R}_{0} \leftarrow \exp (J(\mathbf{w})) \mathbf{R}_{0}$; (5) repeat step (2)(3)(4) until convergence

\section{Optimal Motion Estimation}

A motion $\mathbf{P}$ can be mathematically represented as a rigid transformation (the combination of a rotation $\mathbf{R}$ and a translation $\mathbf{t}$ ) in $\mathbb{R}^{3}$, which corresponds to an element of the Lie group $S E(3)$ (Special Euclidean group). Similar to [11], gradient type method is used in this paper to iteratively adjust rotation and translation parameters. Due to the orthogonality constraint of rotation matrices, unit quaternions are used as the representations of 3D rotations in [11]. However, the applicability of unit quaternions is rather limited because they can only be used in 3D cases. In our algorithm, instead, Lie group $S O(3)$ and its associated Lie algebra $\mathfrak{s o}(3)$ is exploited to provide a novel solution of optimal rotation estimation. One of the virtue of $S O(3)$ representation of rotation is that it can be easily extended to any $n$-dimension case by analogously using $S O(n) . S E(3)$ onmanifold optimization can be straightforwardly achieved by combining 3D translation and $S O(3)$ rotation. The study of $S E(3)$ on-manifold optimization in this paper is very brief, and of course cannot cover the whole field but only the necessary scope used in this paper. Readers can refer to [2] for more details on $S E(3)$ and related optimization.

\section{1. $\mathrm{SO}(3)$ and associated Lie algebra}

A rotation matrix $\mathbf{R}$ within $\mathbf{P}$ is an element of Lie group $S O(3)=\left\{\mathbf{R} \in \mathbb{R}^{3 \times 3}: \mathbf{R}^{T} \mathbf{R}=I,|\mathbf{R}|=1\right\}$, which is referred to as Special Orthogonal group because of its orthogonal characteristic. It has been always an obstacle in rotation-related optimization problems due to the orthogonality constraint. However, recent studies of $S O(3)$ in computer vision and related literature [2][18] reveal that the
$S O(3)$ on-manifold optimization can be used to find appropriate rotation matrices efficiently and elegantly without worrying about the orthogonality constraint. At first, since $S O(3)$ is a Lie group, it should fulfill the associated conditions (and $S E(3)$ as well) [2], and one of them is closure: $\forall \mathbf{R}_{1}, \mathbf{R}_{2} \in S O(3), \mathbf{R}_{1} \mathbf{R}_{2} \in S O(3)$. Secondly, $S O(3)$ is a smooth manifold embedded in $\mathbb{R}^{3}$, which is a topological space wherein all elements are rotation matrices (Figure 2). For each point $\mathbf{R}_{i}$ on the $S O(3)$ manifold, there exists a tangent space, and fortunately, the tangent space of $S O(3)$ happens to be its associated Lie algebra $\mathfrak{s o}(3)$. In other words, $\forall \mathbf{R}_{i} \in S O(3), \exists \Lambda_{i} \in \mathfrak{s o}(3)$. Intuitively, the tangent space of the $S O(3)$ manifold can be understood as a vector space of the derivative of the manifold at point $\mathbf{R}_{i}$ (Figure 2). The mathematical connection between $\mathfrak{s o}(3)$ and $S O(3)$ is:

$\mathfrak{s o}(3) \rightarrow S O(3): \mathbf{R}=\exp (\Lambda), \quad \Lambda \in \mathfrak{s o}(3), \mathbf{R} \in S O(3)$

Lie algebra $\mathfrak{s o}(3)$ is the collection of anti-symmetric matrices, which can be mapped from $\mathbb{R}^{3}$ with a skew operator $J(\cdot)$ defined as:

$$
\mathbf{w}=\left[\begin{array}{l}
w_{1} \\
w_{2} \\
w_{3}
\end{array}\right] \rightarrow J(\mathbf{w})=\left[\begin{array}{ccc}
0 & -w_{3} & w_{2} \\
w_{3} & 0 & -w_{1} \\
-w_{2} & w_{1} & 0
\end{array}\right]
$$

Thus, any point that lies within the infinitesimally small vicinity of a certain point $\mathbf{R}_{0}$ on the $S O(3)$ manifold can be represented as:

$$
\mathbf{R}(\mathbf{w})=\exp (J(\mathbf{w})) \mathbf{R}_{0}
$$

which provides a mapping from vectors in $\mathbb{R}^{3}$ to a local neighboring region of $\mathbf{R}_{0}$ on the $S O(3)$ manifold, and in which the exponential term can be computed using Rodrigues formula:

$\exp (J(\mathbf{w}))=\mathbf{I}+J(\mathbf{w}) \frac{\sin (\|\mathbf{w}\|)}{\|\mathbf{w}\|}+J(\mathbf{w})^{2} \frac{1-\cos (\|\mathbf{w}\|)}{\|\mathbf{w}\|^{2}}$

Finally, in unconstrained optimization problems, gradient type method is popularly used by iteratively updating the solution. Thanks to the local parametrization of $S O(3)$ in (10), we can transform the update of $S O(3)$ to the one with respect to $\mathbb{R}^{3}$ without worrying about the orthogonality constraint. Meanwhile, different from usual cases, instead of computing incremental updates within the same space, in the $S O(3)$ manifold optimization, after every update of w, it needs to be projected back to $S O(3)$. Then, the gradient is computed within the local parametrization of the corresponding neighboring region. The on-manifold optimization scheme of $S O(3)$ is demonstrated in Figure 2.

\subsection{SE(3) on-manifold optimization}

Since the translation corresponds to vectors in $\mathbb{R}^{3}$, with the map from $\mathbb{R}^{3}$ to $S O(3)$ in (10), we can straightforwardly 
establish a map $\Omega$ from $\mathbb{R}^{6}$ to $S E(3)$ manifold as:

$$
\begin{aligned}
& \Omega:[\mathbf{w}, \mathbf{v}]^{\prime} \rightarrow\left\{\exp \left(J(\mathbf{w}) \mathbf{R}_{0}\right), \mathbf{t}_{0}+\mathbf{v}\right\} \\
& \text { s.t. } \quad \mathbf{w}, \mathbf{v}, \mathbf{t}_{0} \in \mathbb{R}^{3}, \mathbf{R}_{0} \in S O(3)
\end{aligned}
$$

Based on the kernel method constructed in section 3, each $3 \mathrm{D}$ point cloud is represented by $\mathrm{KDE}$, which is a distribution in the form of Gaussian mixtures. Thus the rotation and translation on point clouds correspond to rotating and shifting distributions. On one hand, both rotation and translation can affect means of Gaussians; on the other hand, since the covariance is invariant to translation, only rotation will be taken into account. With SVD decomposition of the covariance matrix, we have $\Sigma=U S U^{\prime}$, where $S$ is a diagonal matrix with eigenvalues as diagonal entries, and $U$ is a matrix composed of eigenvectors, so the rotation will only change the orientation of eigenvectors but preserve the magnitude of their corresponding eigenvalues. Thus the KDE of the model point cloud after the Euclidean transformations $\mathbf{P}$ is:

$$
\begin{aligned}
\overline{\phi(x)} & =\mathbf{P} \star \phi(x) \\
& =\frac{1}{m_{\mathcal{M}}} \sum_{i=1}^{m_{\mathcal{M}}} \mathcal{N}\left(x ; \mathbf{P} \lambda_{\mathcal{M}}^{(i)},(\mathbf{R} U) S(\mathbf{R} U)^{\prime}\right) \\
& =\frac{1}{m_{\mathcal{M}}} \sum_{i=1}^{m_{\mathcal{M}}} \mathcal{N}\left(x ; \mathbf{R} \lambda_{\mathcal{M}}^{(i)}+\mathbf{t}, \mathbf{R} \Sigma_{\mathcal{M}}^{(i)} \mathbf{R}^{\prime}\right)
\end{aligned}
$$

Here we abuse notation $\lambda_{\mathcal{M}}^{(i)}$ and $\mathbf{P} \lambda_{\mathcal{M}}^{(i)}$ for both original and homogeneous coordinates because there is no ambiguity. By substituting (13) into (5)(6), the objective function is the kernel function between $\mathcal{M}$ transformed with $\{\mathbf{R}, \mathbf{t}\}$ and $\mathcal{S}$ :

$$
\begin{aligned}
& K\left(\mathcal{M}_{\mathbf{R}, \mathbf{t}}, \mathcal{S}\right)=K_{\exp }(\bar{\phi}(x), \psi(x)) \\
&=\frac{1}{m_{\mathcal{M}}} \frac{1}{m_{\mathcal{S}}} \sum_{i=1}^{m_{\mathcal{M}}} \sum_{j=1}^{m_{\mathcal{S}}} \mathcal{G}_{\mathcal{M}_{\mathbf{R}, \mathbf{t}}^{i} \mathcal{S}^{j}} \\
& \mathcal{G}_{\mathcal{M}_{\mathbf{R}, \mathbf{t}}^{i} \mathcal{S}^{j}}=\frac{1}{(2 \pi)^{3 / 2}\left|\mathbf{G}_{i j}\right|^{1 / 2}} \exp \left(-\frac{1}{2} \Delta_{i j}{ }^{\prime} \mathbf{G}_{i j}{ }^{-1} \Delta_{i j}\right)
\end{aligned}
$$

where $\Delta_{i j}=\lambda_{\mathcal{S}}^{(j)}-\mathbf{R} \lambda_{\mathcal{M}}^{(i)}-\mathbf{t}$ and $\mathbf{G}_{i j}=\mathbf{R} \Sigma_{\mathcal{M}}^{(i)} \mathbf{R}^{\prime}+\Sigma_{\mathcal{S}}^{(j)}$. Then the optimal motion can be estimated by maximizing the kernel function (14):

$$
\left\{\mathbf{R}^{*}, \mathbf{t}^{*}\right\}=\arg \max _{\mathbf{R}, \mathbf{t}} \underbrace{K\left(\mathcal{M}_{\mathbf{R}, \mathbf{t}}, \mathcal{S}\right)}_{\mathbf{O}}
$$

By substituting (12) into (16), the objective function $\mathbf{O}$ is dependent only on $\mathbf{w}$ and $\mathbf{v}$. The gradient can be computed as (check supplementary material for detailed derivation):

$$
\begin{gathered}
\frac{\partial \mathbf{O}}{\partial \mathbf{v}}=\frac{1}{m_{\mathcal{M}} m_{\mathcal{S}}} \sum_{i=1, j=1}^{m_{\mathcal{M}}, m_{\mathcal{S}}} \mathcal{G}_{\mathcal{M}_{\mathbf{R}, \mathbf{t}}^{i} \mathcal{S}^{j}} \Delta_{i j}^{\prime} \mathbf{G}_{i j}^{-1} \\
\frac{\partial \mathbf{O}}{\partial \mathbf{w}}=\sum_{i=1, j=1}^{m_{\mathcal{M}}, m_{\mathcal{S}}}\left\{\left(\frac{1}{2}\left(\Delta_{i j}^{\prime} \mathbf{G}_{i j}^{-1}\right) \otimes\left(\Delta_{i j}^{\prime} \mathbf{G}_{i j}^{-1}\right)-\operatorname{vec}\left(\mathbf{G}_{i j}^{-1}\right)^{\prime}\right)\right. \\
\left.\cdot \frac{\partial \mathbf{G}_{i j}}{\partial \mathbf{w}}-\Delta_{i j}^{\prime} J\left(\mathbf{R}_{n} \lambda_{m}^{(i)}\right)\right\} \mathcal{G}_{\mathcal{M}_{\mathbf{R}, \mathbf{t}}^{i} \mathcal{S}^{j}}
\end{gathered}
$$

Where $\operatorname{vec}(\cdot)$ operator vectorizes a matrix by stacking its columns, and $\frac{\partial \mathbf{G}_{i j}}{\partial \mathbf{w}}$ is:

$$
\frac{\partial \mathbf{G}_{i j}}{\partial \mathbf{w}}=\left(\mathbf{I}_{9}+T_{3,3}\right)\left(\mathbf{R} \boldsymbol{\Sigma}_{\mathcal{M}}^{(i)} \boldsymbol{\Sigma}^{\prime} \otimes \mathbf{I}_{3}\right) \frac{\partial J(\mathbf{w})}{\partial \mathbf{w}}
$$

where $\mathbf{I}_{n}$ is a $n \times n$ identity matrix, $T_{3,3}$ is a permutation matrix which satisfies $T_{3,3} \operatorname{vec}(\mathbf{A})=\operatorname{vec}\left(\mathbf{A}^{\prime}\right)$ for any $3 \times$ 3 matrix, $\frac{\partial J(\mathbf{w})}{\partial \mathbf{w}}$ can be easily computed according to the definition (9).

\section{Experiments}

With the Jacobian vector $\left\{\nabla_{\mathbf{w}}, \nabla_{\mathbf{v}}\right\}$ computed in (17)(18), a gradient descent method for point cloud registration can be summarized as Algorithm 1.

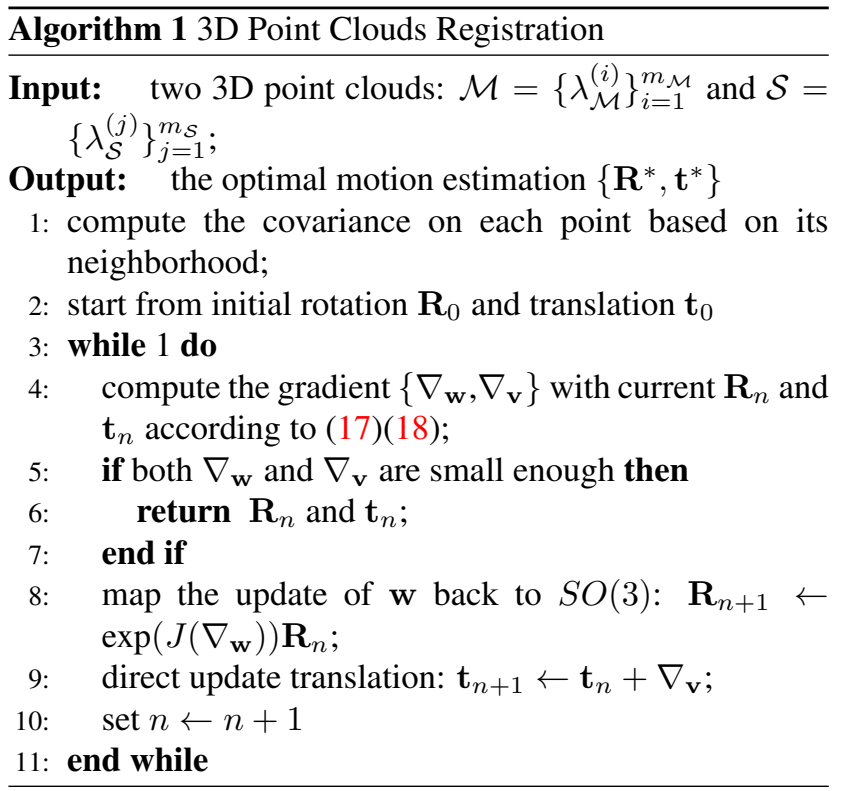

\subsection{Qualitative Experiments}

At first, we test our algorithm qualitatively on KIT 3D database[13]. Since each 3D object model in the database is in triangulated mesh format, we generate the corresponding point cloud by first sampling a triangle with the probability proportional to its area and then uniformly sampling a point within the selected triangle. For each point cloud (red points in Figure 3), a random motion is generated and applied on it. In addition, either random outliers are added or random part of object are removed to generate a synthetic target point cloud (blue points in Figure 3). Some test results of our registration algorithm is displayed in Figure 3. We can see that the proposed algorithm can work qualitatively well in many challenging cases (registration results in green frames). However, the algorithm will also fail when the rotation angle is bigger than $90^{\circ}$ or due to the overlarge 

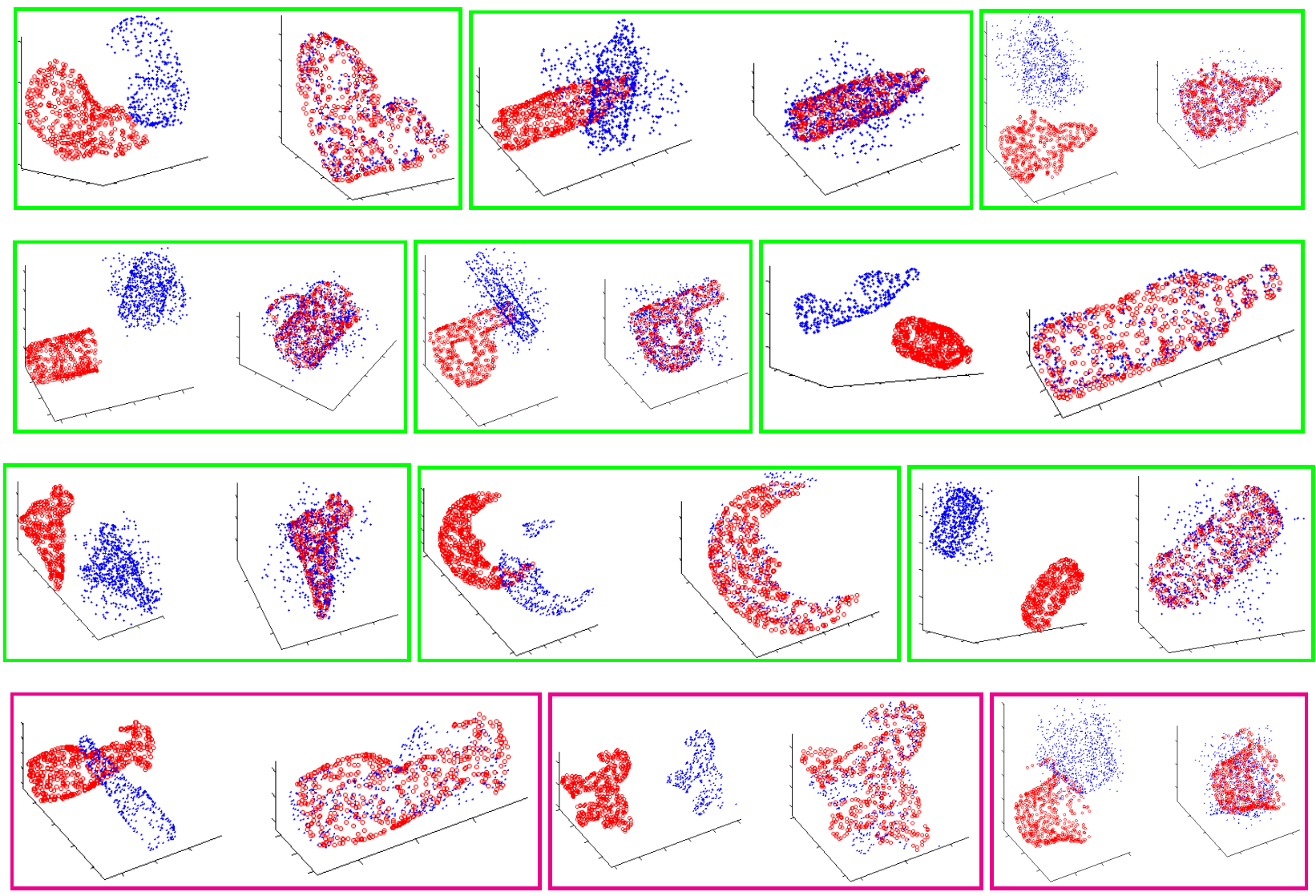

Figure 3. A sample set of qualitative test of the proposed algorithm on KIT database.

amount of outliers or missing points (registration results in magenta frames). The instability of the performance stems from the fact that the algorithm is likely to stuck into local optimum.

\subsection{Quantitative Experiments}

To obtain a more precise evaluation of the proposed algorithm, we conduct several quantitative experiments with different motion scales, outliers and missing portions for test. In addition, we also implement and run ICP and Jian's method [11] for comparison. To ensure the fairness of the comparison, the same $S E(3)$ optimization strategy and stop criterion are used for their corresponding objective functions. KIT database is used as well here for quantitative evaluation and the point clouds are generated in the same way as section 5.1.

First, we test the robustness of three algorithms on different scales of motions. In this experiment, for motion scale $i$, the rotation angles of yaw, pitch and roll are $i \times\left[20^{\circ}, 4^{\circ}, 4^{\circ}\right]$, and translations are $i \times\left[S_{x}, S_{y}, S_{z}\right]$, where $\left[S_{x}, S_{y}, S_{z}\right]$ are standard deviations of point clouds in three axes. Different motions are applied on the point cloud of each object (points cloud is sampled with size 1000) to generate a tar- get point cloud to align with. Since we know the correspondence between the original and target point clouds, the error for each registration is computed as the average distance between every pair of corresponding points in two point clouds. The test result on the database is plotted in Figure 4(a). We can see that ICP method is very sensitive to the initial motion. Actually average error of ICP method is monotonic increasing with respect to motion scale. Jian's method and our method work in almost the same pattern. Both method work rather accurately when the motion scale $i \leq 4$ (rotation angle is smaller than $90^{\circ}$ ). However, both methods will fall into the local minimum (reflectional symmetry pose) when the motion scale increase ever since.

Secondly, we test the robustness of three algorithms by adding different portion (the percentage of point cloud size) of outliers. Outliers are generated by randomly sampling within the space around objects. For portion $i$, the number of added outliers is $i \times 10 \%$ of the point-cloud size. The generated outliers are concatenated into the original point clouds, so the correspondence ground-truth is still available and the registration error is computed in the same way as in the motion experiment. To avoid the effect of large motion, a relatively small motion (motion scale $i=1$ ) is ap- 


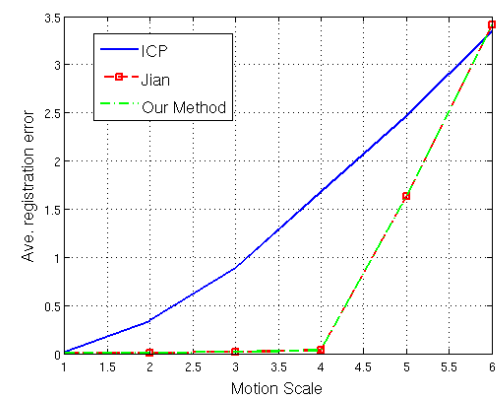

(a)

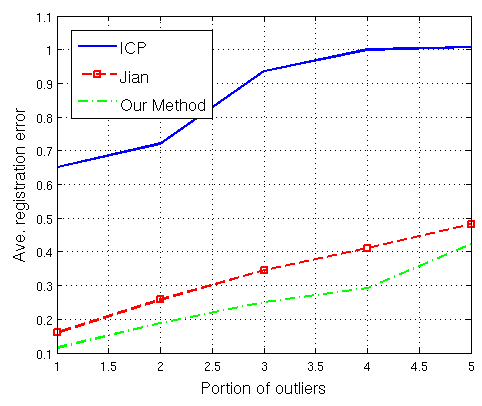

(b)

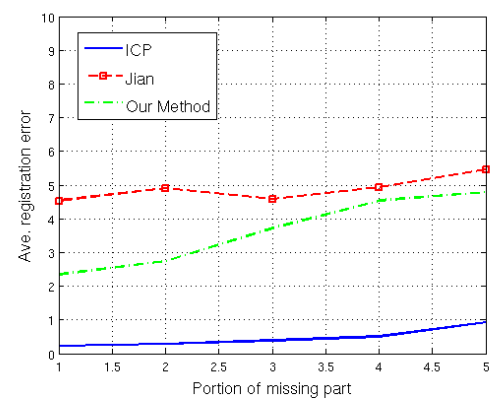

(c)

Figure 4. Performance comparison of three registration methods on the KIT database with different motion scales, portion of outliers and portion of missing part respectively.

plied on point clouds. The test result is plotted in Figure 4(b), from which we can see that ICP method is very fragile when the outliers are present even the amount is small. By contrast, since structure of point clouds are globally modelled in Jian's method and our method, they are much more robust to the presence of outliers. In addition, due to the local estimated covariance in our method, it can outperform Jian's method for all portion $i$.

Finally, part missing is an usual case because of occlusion. Here we also compare the performance of three algorithms on different portion of missing part. A missing part is selected by first randomly picking a point and then removing all points which lie within the neighbourhood of certain range. For portion $i$, the number of eliminated outliers is $i \times 6 \%$ of the point-cloud size. Since the seed point is randomly selected, the correspondence ground truth will not be available between the original point cloud and target partial point cloud. To measure the registration accuracy, the full target point cloud is preserved before a random part is removed, and the registration error is computed as the distance between full original point cloud and full target point cloud. Similarly, a relatively small motion (motion scale $i=1$ ) is applied on point clouds to avoid the effect of large motion. The test result is displayed in Figure 4(c). It can be seen that the performance of ICP method is most stable in missing cases. As for Jian's method and our method, unfortunately, the global structure will be greatly changed when a random part is removed, neither of their performance is acceptable although our method is better than Jian's method by making use of local structure information embedded in the local estimated covariance. However, it should be reminded that ICP method can outperform two others in missing cases only under the condition that the initial motion is small.

\section{CONCLUSION}

We present a full study on how probability product kernel function can be used for 3D point cloud registration. A general registration framework is developed by incorporating $S E(N)$ on manifold optimization strategy. According to empirical test, the proposed registration algorithm is accurate and robust in many challenging cases. However, even with the help of local estimated covariance, the performance of our algorithm is still unacceptable when certain part of point cloud is missing, which points to the future direction of our research.

\section{References}

[1] P. J. Besl and H. D. Mckay. A method for registration of 3-D shapes. PAMI, 14(2):239-256, 1992. 1, 2

[2] J. L. Blanco. A Tutorial on SE(3) Transformation Parameterizations and on-Manifold Optimization. Technical report, University of Malaga, Sept. 2010. 4

[3] M. Carcassoni and E. R. Hancock. Spectral Correspondence for Point Pattern Matching. Pattern Recognition, 36(1):193204, 2003. 2

[4] D. Chetverikov, D. Svirko, D. Stepanov, and P. Krsek. The Trimmed Iterative Closest Point Algorithm. In ICPR, pages 545-548, 2002. 2

[5] R. Detry and J. Piater. Continuous surface-point distributions for $3 \mathrm{D}$ object pose estimation and recognition. In $A C C V$, volume 6494 of $L N C S$, pages 572-585, Heidelberg, 2010. Springer. 3

[6] R. Detry, N. Pugeault, and J. Piater. A Probabilistic Framework for 3D Visual Object Representation. PAMI, 31(10):1790-1803, 10 2009. 3

[7] J. Feldmar, N. Ayache, and F. Betting. 3D-2D projective registration of free-form curves and surfaces. Computer Vision and Image Understanding, 65:403-424, 1997. 2

[8] G. Godin, M. Rioux, and R. Baribeau. Three-dimensional registration using range and intensity information. In Proceedings of the SPIE: Videometrics III, volume 2350, pages 279-290, Boston, Massachusetts, USA, Nov. 1994. 2

[9] S. Gold, A. Rangarajan, C. ping Lu, and E. Mjolsness. New Algorithms for 2D and 3D Point Matching: Pose Estimation and Correspondence. Pattern Recognition, 31:957-964, 1997. 2 
[10] T. Jebara, R. Kondor, and A. Howard. Probability Product Kernels. Journal of Machine Learning Research, 5:819-844, July 2004. 3

[11] B. Jian and B. C. Vemuri. Robust Point Set Registration Using Gaussian Mixture Models. PAMI, 33(8):1633-1645, 2011. 2, 3, 4, 6

[12] A. Johnson and S. B. Kang. Registration and Integration of Textured 3-D Data. In Image and Vision Computing, pages 234-241, 1996. 2

[13] A. Kasper, Z. Xue, and R. Dillmann. The KIT object models database: An object model database for object recognition, localization and manipulation in service robotics. The International Journal of Robotics Research, May 2012. 5

[14] R. Kondor and T. Jebara. A kernel between sets of vectors. In ICML, 2003. 3

[15] Y. Liu, L. Li, and B. Wei. 3D shape matching using collinearity constraint. In ICRA, pages 2285-2290, 2004. 2

[16] S. Lyu. A Kernel Between Unordered Sets of Data: The Gaussian Mixture Approach. In ECML, pages 255-267, 2005. 3

[17] T. Pajdla and L. V. Gool. Matching of 3-D Curves using Semi-differential Invariants. In ICCV, pages 390-395. IEEE Computer Society Press, 1995. 2

[18] M. D. Plumbley. Lie group methods for optimization with orthogonality constraints. In Int. Conf. on Independent Component Analysis and Blind Signal Separation, pages 12451252. Springer, 2004. 4

[19] C. E. Rasmussen and C. Williams. Gaussian Processes for Machine Learning. MIT Press, 2006. 3

[20] S. Rusinkiewicz and M. Levoy. Efficient Variants of the ICP Algorithm. In 3DIM, pages 145-152, 2001. 2

[21] R. B. Rusu, Z. C. Marton, N. Blodow, and M. Beetz. Persistent Point Feature Histograms for 3D Point Clouds. In Int. Conf. on Intelligent Autonomous Systems, page 477. 2008. 2

[22] J. Shawe-Taylor and N. Cristianini. Kernel methods for pattern analysis. Cambridge University Press, 2004. 2

[23] R. Yang and P. K. Allen. Registering, Integrating, and Building CAD Models from Range Data. In ICRA, pages 31153120, 1998. 2

[24] L. Zhang, S.-I. Choi, and S.-Y. Park. Robust ICP Registration Using Biunique Correspondence. In 3DIMPVT, pages 8085. IEEE, 2011. 2

[25] Z. Zhang. Iterative point matching for registration of freeform curves and surfaces. International Journal of Computer Vision, 13(2):119-152, 1994. 2 